\title{
A SUMMARY OF THE GPS SYSTEM PERFORMANCE FOR STARS MISSION 3
}

\author{
Earl E. Creel, Senior Member of Technical Staff \\ Sandia National Laboratories \\ Albuquerque, New Mexico
}

RECEIVED

JUL 171997

\begin{abstract}
This paper describes the performance of the GPS system on the most recent flight of the STARS missile, STARS Mission 3 (M3). This mission was conducted under the Ballistic Missile Defense Organization's (BMDO's) Consolidated Targets Program. The United States Army Space and Strategic Defense Command (USASSDC) is the executing agent for this mission and the Department of Energy's (DOE's) Sandia National Laboratories (SNL) is the vehicle developer and integrator. The M3 flight, dually designated as the MSX Dedicated Targets II (MDT-II) mission occurred on August 31, 1996. This mission was conducted for the specific purpose of providing targets for viewing by the MSX satellite. STARS M3 was the first STARS flight to use GPS-derived data for missile guidance, and proved to be instrumental in the procurement of a wealth of experimental data which is still undergoing analysis by numerous scientific agencies within the BMDO complex. GPS accuracy was required for this mission because of the prescribed targeting requirements for the MDT-II payload deliveries with respect to the MSX satellite flight path. During the flight test real time GPS-derived state vector data was also used to generate pointing angles for various down range sensors involved in the experiment. Background information describing the STARS missile, GPS subsystem architecture, and the GPS Kalman filter design is presented first, followed by a discussion of the telemetry data records obtained from this flight with interpretations and conclusions.
\end{abstract}

\section{The STARS Missile}

The Strategic Target System (STARS) missile program is sponsored by the United States Army Space and Strategic Defense Command (USASSDC). The system is an ICBM-class missile and is intended as a means of low cost target delivery to the Kwajalein area in support of Ballistic Missile Defense
Organization (BMDO) programs ${ }^{[1]}$. The system front section is designed, assembled, and tested by Sandia National Laboratories (SNL), Albuquerque, NM, and Livermore, CA. Each missile is assembled and pad-launched by SNL from DOE's Kauai Test Facility (KTF) at Barking Sands, Kauai, HI.

The STARS missile has two primary configurations designated as STARS I and STARS II. Both configurations have three solid rocket motor stages, consisting of refurbished Polaris $A 3$ motors for the first and second stage; and a third stage ORBUS 1 motor which was developed specifically for the STARS missile. The STARS II configuration has an additional fourth stage Post Boost Vehicle (PBV) which has maneuvering and acceleration capabilities ${ }^{[2]}$. The PBV is powered by liquid hypergolic propellants and contains all navigation system components and most all other electronic components necessary to completely fly the missile. Several target objects can be deployed from a payload plate that sits on top of the PBV ${ }^{[3]}$. Four STARS missions have occurred to date beginning with a STARS I Flight Test Development Unit (FTU-1), in February 1993, followed by 3 operational missions designated $M 1, M 2$, and $M 3$.

The STARS GPS system was first flown in August 1993 on the STARS Mission 1 (M1) flight (STARS I configuration). The primary goal of this flight was to demonstrate the capability to maintain continuous GPS Precise Positioning Service (PPS) accuracy from liftoff through reentry, including periods of high vehicle dynamics Motivation for this development stemmed from a curiosity of whether the primary goal was achievable coupled with a desire to enhance the missile

UNCLASSIFIED - Approved for Public Release; Distribution is Unlimited

Sandia is a multiprogram laboratory operated by Sandia Corporation, a Lockheed Martin Company, for the United States Department of Energy under Contract DE-AC04-94AL85000. 


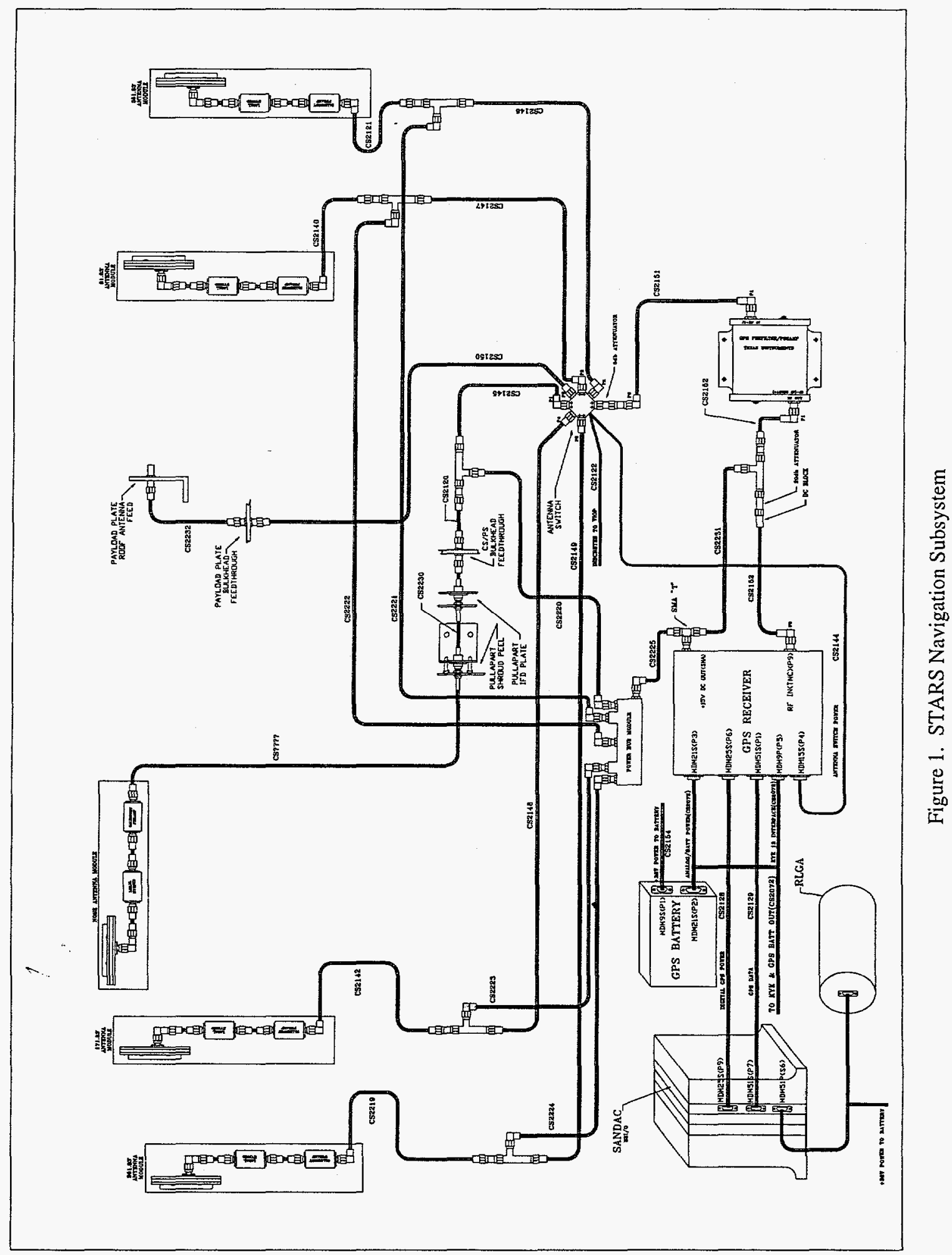


targeting accuracy for payload deliveries on future flights. With the GPS capability comes the additional benefits of accurate real-time scoring data and auxiliary range safety information. The success of the STARS M1 GPS system subsequently laid the groundwork for more aggressive use of the capability on the STARS M2 and M3 (both STARS II) flights.

\section{Inertial Navigation System}

Figure 1 illustrates the STARS navigation subsystem which includes a ring laser gyrobased strapdown inertial measurement unit (IMU) known as the Ring Laser Gyro Assembly (RLGA). The RLGA was jointly developed by SNL and Honeywell and is based on the Honeywell 1320 ring laser gyro. The RLGA can be optionally configured with a single-axis roll gimbal making it ideal for use in spinning vehicle applications. The STARS flight computer is a multi-processor SNLdesigned component known as the SANDAC V (SANdia Airborne Computer) ${ }^{[4]}$. The design is modular and rugged and can be configured for use in applications having severe vibration and shock environments. Current SANDAC computers are based on the Motorola MC68040 microprocessor. Up to 16 processor cards can exist in a SANDAC stack. Numerous other peripheral boards such as 16-bit parallel I/O, high speed serial, and MIL-STD 1553B are also available for connecting SANDAC to a wide variety of inertial navigation systems, GPS receivers, and host vehicle interfaces. All navigation guidance and control functions, including strapdown navigation calculations and GPS Kalman filter algorithms are performed by SANDAC. The STARS M3 SANDAC is a six module configuration which includes three processor modules; one MIL-STD 1553B module; a single "smart" system input/output module; and a power supply card referred to as the "utility module", which also hosts additional other system resources

\section{GPS Receiver}

Texas Instruments (TI) is the vendor for the STARS GPS receiver referred to as the GPS Embedded Module (GPSEM). The receiver simultaneously tracks $\mathrm{P}(\mathrm{Y})$-code from up to six satellites on either $L_{1}$ or $L_{2}$. Each tracker channel has its own dedicated hardware which is based on TI's proprietary channel-ona-chip technology. During normal operation the receiver tracks $L_{1}$ on five channels while tracking $L_{2}$ signals from the same set of five SV's on the sixth channel in a roving fashion. The receiver module is compact in size ( $<90 \mathrm{cu}$ in) and consumes less than $15 \mathrm{~W}$ of power. The GPSEM has an instant-on oscillator and also contains the Magnavox PPSSM security module which supports the KYK-13 interface specification for the loading of cryptographic key materials. The GPSEM was originally designed to reside inside the card cage of a Honeywell SCINS navigation system and communicate with the SCINS computer through a dual port memory interface. Since the GPSEM lacked sufficient mechanical mounting, SNL designed a selfcontained enclosure for the receiver complete with data bus and power interfaces. This enclosure design has evolved to include integral mechanical shock isolators and a pressure seal. The seal eliminates rapid changes in the ambient pressure which the receiver clock otherwise experiences during missile ascent.

\section{GPS Antenna System}

The STARS M3 antenna system depicted in Figure 1 uses 5 discrete patch antenna elements designed by the SNL antenna group. The elements consist of "stacked" thin copper patches, one each for the $L_{1}$ and $L_{2}$ frequency band, which are adhered to a ceramic/teflon substrate. The ceramic piece is captured in a lightweight aluminum frame and a teflon protective cover is placed over the top. The feeds from the patches are combined externally using a commercially supplied combiner and semi-rigid RF cable. To enhance the system noise figure each antenna element has a local Salisbury preamplifier ( $26 \mathrm{~dB}$ of gain) and integral bandpass filters centered at the $L_{1}$ and $L_{2}$ frequencies. One of the five hemisphericalpattern antenna elements mounts in the nose tip of the ascent shroud. The other four are mounted around the perimeter of the PBV (with polar axis perpendicular to the missile skin) at a vertical position aligned with an RFtransparent window in the ascent shroud ${ }^{[5]}$. 
Table 1. STARS M3 GPS Antenna Assignments

\begin{tabular}{|c|l|l|}
\hline Antenna ID & \multicolumn{1}{|c|}{ Location } & \multicolumn{1}{|c|}{ Mission Usage } \\
\hline 1 & shroud nose tip & $\begin{array}{l}\text { pre-launch } \\
1^{\text {st }} \text { stage climb out } \\
\text { earl } 2^{\text {nd }} \text { stage }\end{array}$ \\
\hline 2 & PBV 0 deg & PBV powered flight \\
\hline 3 & PBV 90 deg & PBV powered flight \\
\hline 4 & PBV 180 deg & PBV powered flight \\
\hline 5 & PBV 270 deg & $\begin{array}{l}\text { late } 2^{\text {nd }} \text { stage } \\
3^{\text {rd }} \text { stage } \\
\text { PBV powered flight }\end{array}$ \\
\hline 6 & payload plate area & ground test \\
\hline
\end{tabular}

Table 1 lists the antenna number assignments and physical locations.

A 6-input Macom Omni-Spectra RF pin-diode switch is used to connect the antenna elements one-at-a-time to a TI-designed cavity-backed (20 dB gain) preamplifier feeding the receiver input. The sixth spare input is cabled to an RF connector accessible through the skin of the missile in the payload area. This port is connected to a facility antenna for ground testing when the missile is on the launch pad and enclosed by the missile service tower. The flight computer commands the pin diode switch to connect the receiver to a particular antenna element depending on the attitude of the vehicle. Prior to liftoff and then during the first stage burn, the shroud nose antenna is used since the missile is in a nearly vertical attitude. As the missile pitches over during the second stage burn, SANDAC commands the RF switch to divert to the antenna having the best upwards look. During the third stage burn the missile pitch and roll angles remain fairly constant, so no additional antenna switching occurs. After PBV separation there is frequent antenna switching due to changes in vehicle attitude as the mission profile is executed. Any of the four antennas mounted on the PBV can be used depending on which one has the best look at the GPS satellites above.

\section{Receiver Tracking Performance}

Telemetry records indicate that the receiver tracked near the typical maximum (5) SV'S for almost the entire flight. The GPSEM reports status information for each tracker channel, including the track loop state. As the GPSEM data arrives each second, the flight computer tallies how many of the tracker channels have combined good overall state-of-health and the required track status. Each second this tally can range in value between 0 and 6 . Figure 2 illustrates the actual number of SV's tracked verses time of flight. Note that the system performs best when four or more sets of measurements are available.

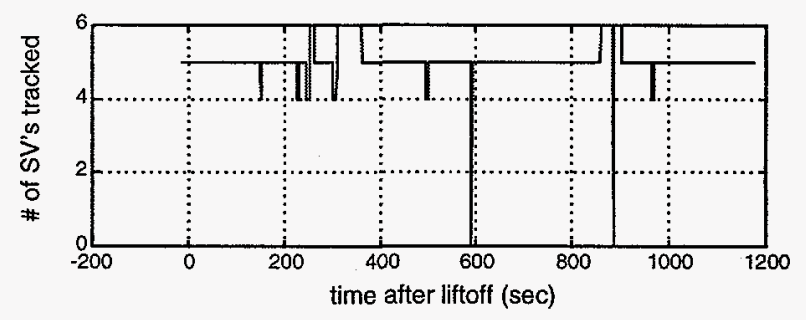

Figure 2. Plot of the number of SV's tracked (phaselock Y-code) verses time of flight

A counter was mechanized in the software to increment at a $1 \mathrm{~Hz}$ rate for each time the receiver indicated that four or more SV data measurement sets were valid. This counter value is plotted in Figure 3. Out of 1172 total seconds of TM data the counter incremented a total of 1170 times. This implies that the receiver tracked four or more SV's $99.8 \%$ of the time between missile liftoff and reentry. The actual tracking percentage may of been somewhat less than this, however, due to the fact that there are about $20 \mathrm{~s}$ of randomly

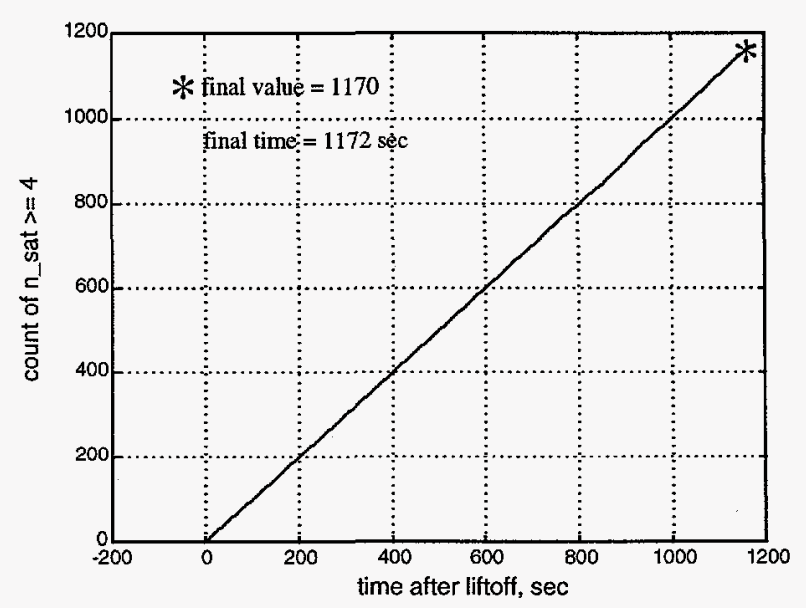

Figure 3. Plot of the number of times that 4 or more SV's were tracked in flight 
distributed TM data points missing from the mission timeline due to dropouts. If all of the missing data points were associated with degraded tracking (which is highly unlikely) the receiver still tracked four or more SV'S $98.1 \%$ of the time.

Figure 4 illustrates that all five GPS antennas were used at various times throughout the M3 mission. At about $500 \mathrm{~s}$ into the flight, the PBV performed a significant attitude maneuver causing the system to switch between four different antennas in a relatively brief time span. Interestingly, the number of SV's tracked by the receiver during this time period remained almost constant, dipping momentarily from five to four (see Figure 2).

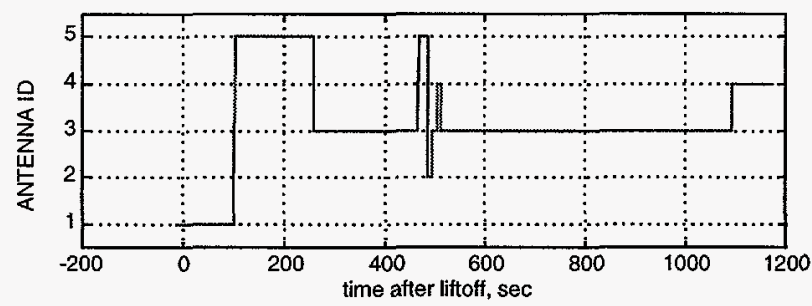

Figure 4. Antenna switching history

Position Dilution of Precision (PDOP) and Geometric Dilution of Precision (GDOP) were both good for the entire flight as shown in Figure 5. This result stems directly from having full operational status for the Block II GPS SV constellation and allows the issue of

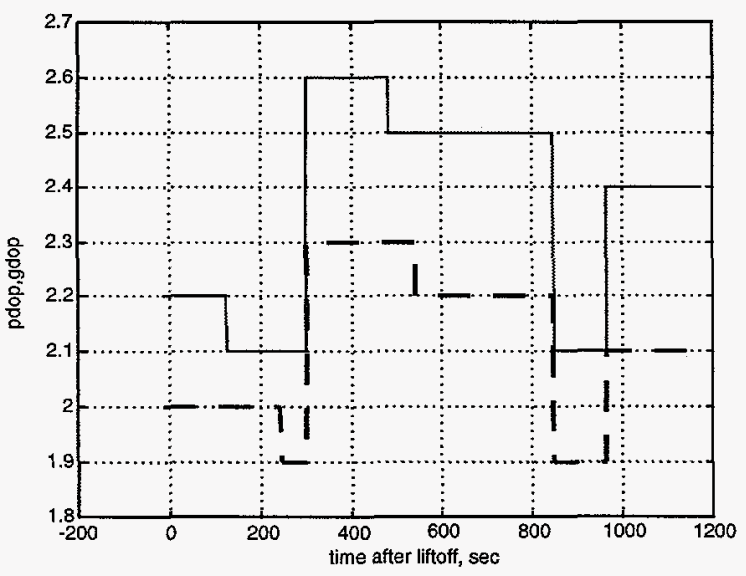

Figure 5. PDOP (dashed) and GDOP (solid) as reported in flight by the GPSEM
SV visibility for these kind of applications to be virtually ignored. High altitudes of operation coupled with an abundance of SV signals almost guarantees good to great DOP values for operating regions in the low to middle latitude regions of the globe. Momentary DOP anomalies do not affect the STARS GPS system performance in general because of the way that the measurement data are processed on an "any and allavailable basis".

Table 2 summarizes the SV tracking history for each of the GPSEM tracker channels. A total of 11 different SV's were tracked during the mission at average carrier-to-noise density ratios ranging from 34.3 to 47.2 . Channels 3 and 5 tracked a single SV for the entire $2000^{+}$mile trajectory, and the largest number of different SV's tracked by any single channel was three. At liftoff, channel 2 was the rover. The rover later switched to channel 6 between $245 \mathrm{~s}$ and $360 \mathrm{~s}$ into the flight and continued to reliably track $L_{2}$ signals for the duration of the mission.

Table 2. Tracking History for all receiver channels

\begin{tabular}{|c|c|c|c|c|c|}
\hline $\begin{array}{c}\text { Receiver } \\
\text { Ch \# }\end{array}$ & SV PRN & talo range & $\begin{array}{c}\text { SV Azimuth } \\
\text { range (deg) }\end{array}$ & $\begin{array}{c}\text { SVElevation } \\
\text { range (deg) }\end{array}$ & $\begin{array}{c}\text { Average } \\
\text { C/No }\end{array}$ \\
\hline \multirow{2}{*}{1} & 1 & $0: 882$ & $235: 215$ & $9: 24$ & 40.2 \\
\cline { 2 - 6 } & 3 & $883:$ reentry & $249: 245$ & $10: 20$ & 41.2 \\
\hline \multirow{2}{*}{2} & Rover & $0: 244$ & n/a & n/a & n/a \\
\cline { 2 - 6 } & 9 & $245: 300$ & 154 & 5 & 34.3 \\
\cline { 2 - 6 } & 5 & $301: 963$ & $101: 97$ & $24: 6$ & 37.7 \\
\cline { 2 - 6 } & 25 & $964:$ reentry & $318: 328$ & $57: 32$ & 45.4 \\
\hline 3 & 6 & $0:$ reentry & $355: 26$ & $51: 27$ & 44.9 \\
\hline 4 & 10 & $0: 244$ & $64: 62$ & $10: 5.7$ & 38.9 \\
\cline { 2 - 7 } & 16 & $245: 300$ & 68 & 6 & 36.1 \\
\hline 5 & 23 & $301:$ reentry & $168: 145$ & $9: 11$ & 40.8 \\
\hline 6 & 17 & $0:$ reentry & $197: 107$ & $57: 65: 59$ & 47.2 \\
\hline & 28 & $0: 360$ & $274: 277$ & $22: 28$ & 39.9 \\
\cline { 2 - 6 } & Rover & $361:$ reentry & n/a & n/a & n/a \\
\hline
\end{tabular}

GPS Software Implementation

GPS related flight software is partitioned into three major pieces; (1) the extended Kalman filter; (2) the inertial track loop aiding software; and (3) the steering loop control logic which closes the guidance loop using the GPS-derived navigation solution.

\section{Extended Kalman Filter}

One piece of the GPS software implements a 13-state extended Kalman filter which 
executes at the $1 \mathrm{~Hz}$ GPS measurement rate. The filter processes PR and PR-dot measurements to estimate the following: INS latitude, longitude, and altitude errors; INS north, east, and down velocity errors; INS north, east, and down attitude errors; and direct estimates of bias, drift, drift-dot, and gsensitive drift for the receiver clock. Measurements are processed $\mathrm{N}$ sets at-atime, $N$ ranging from 1 to 6 , for all measurements marked valid by the receiver.

The Kalman filter implementation is illustrated in Figure 6. Measurement updates and filter gain calculations are both done at a $1 \mathrm{~Hz}$ rate. A receiver measurement set is discarded if it is more than 6-sigma different from the extrapolated measurement prediction formed from the most recent set of error estimates. These differences, referred to as "residuals" provide direct measures of how well the corrected INS solution (raw INS + filter error estimates) agrees with the predicted position and velocity based on GPS measurement data. If several successive measurement sets are discarded the control logic automatically begins to increase the filter covariance values allowing the estimates to change by the required amounts so that the measurement residuals fall within the 6-sigma limits.

\section{Inertial Track Loop Aiding}

The track loop aiding software executes at the $64 \mathrm{~Hz}$ rate of the INS. The code in this loop generates receiver code and carrier track loop aiding data which includes: position, velocity, acceleration, attitude, body rate, and antenna lever arm data. This same loop generates an additional set of aiding data which is passed to the receiver once per second containing such things as receiver clock bias and drift rate estimates. Properly coordinitized aiding data is required to achieve robust performance from the tracking loops, especially during the motor burns when the missile experiences high jerk levels and achieves sustained acceleration levels in excess of $6 \mathrm{~g}^{\prime} \mathrm{s}$.

\section{Missile Steering Loop Control Logic}

Flight software for the STARS M3 mission was configured with the goal of maximizing the

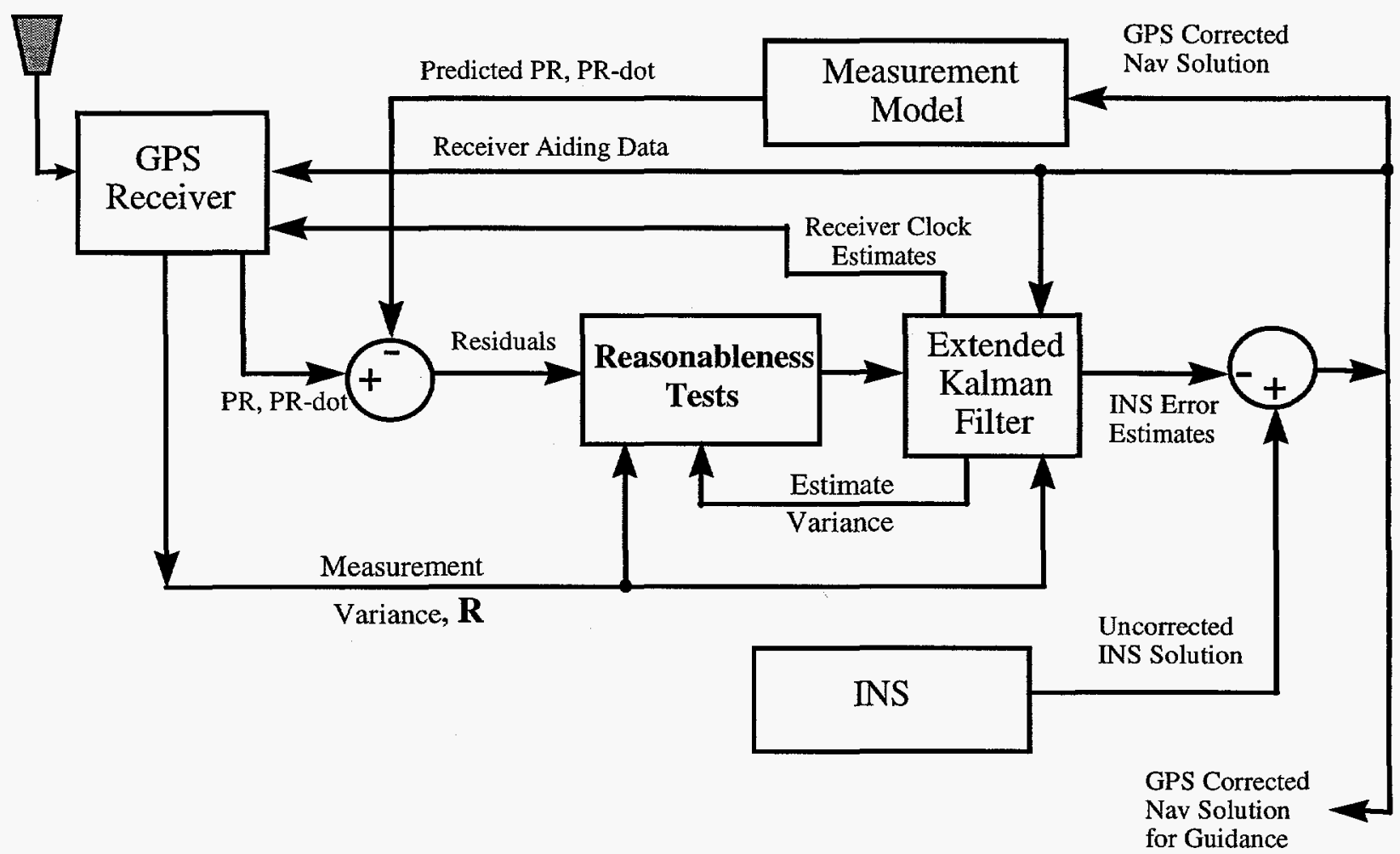

Figure 6. GPS Kalman Filter Mechanization 
probability of mission success. Many of the algorithms which dictate how the system behaves in flight were coded to fail gracefully and predictably in the presence of bad or nonexistent sensor data. The logic which governs "when" and "how" GPS filter data is used to steer the missile was implemented from this perspective.

Past experience created concerns about the quality of the GPS measurement data during first stage climb out. During the July 1994 STARS Mission 2 flight, the clock on a similar GPS receiver experienced a significant change in drift rate as the missile ascended through the atmosphere. This clock change, illustrated in Figure 7, generated very large

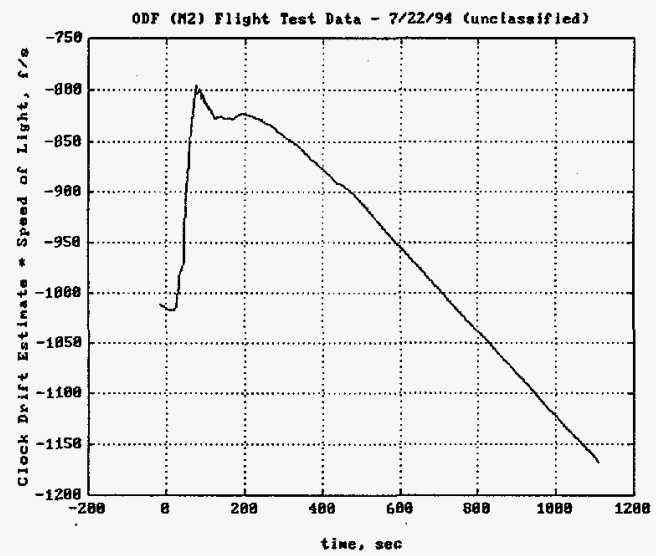

Figure 7. Receiver clock drift rate estimate on STARS M2

filter residuals, and for a time, caused the filter to reject all of the receiver measurement data. Eventually the filter logic "opened-up" the associated covariances far enough that the filter recovered, but, several tens of seconds were required to do so, and in the meantime the filter error estimates experienced degraded fidelity. Shortly after second stage ignition the missile system became exoatmospheric and the receiver clock from that point on maintained consistent performance. Subsequent testing in an altitude chamber with replica receiver hardware verified that there was a definite relationship between rapid atmospheric pressure changes and the receiver's clock drift rate. To address this problem for the $M 3$ mission the enclosure for the GPS receiver was redesigned so that it could be sealed. Preflight altitude chamber testing revealed that this seal was not completely air tight, however, it slowed the leak rate sufficiently so that the character of the clock drift rate remained consistent and predictable.

Since the STARS IMU contains very high quality navigation sensors it does not incur significant errors during the early stages of flight. System accuracy is good enough to satisfy range safety requirements during first stage climb-out without the use of GPS corrections. Errors that do accumulate during the first stage burn can easily be compensated for later by the second and third stage guidance algorithms. ${ }^{[6]}$ GPS filter estimates were not used during the first stage burn in order to avoid the potential problems associated with the receiver's clock pressure sensitivity during atmospheric ascent.

The first stage on the STARS missile fires for approximately 60 s and burnout occurs at an altitude of approximately $100 \mathrm{kft}$. The second stage then fires immediately and burns for 80s. The operating scenario for the missile beyond second stage is tailored specifically for each mission. For STARS M3 there was a brief coast period after second stage burnout at which time the ascent shroud was jettisoned. Next, a small cold gas attitude maneuver was performed, followed immediately by third stage ignition. After third stage burnout another small cold gas maneuver was performed and then the PBV was released in preparation for target deployments.

During the initial part of the second stage burn the onboard steering control loop logic began the process of deciding whether to close the steering loop using INS error estimates from the GPS Kalman filter. The algorithm for determining "if" and "when" to close the steering loop was implemented as follows:

1. Mission time must be between $T+60$ and $T+175$ seconds (corresponds to second stage ignition through third stage burnout) 
2. While inside the time-window-ofopportunity, the following must occur simultaneously:

(a) Ten (10) $1 \mathrm{~Hz}$ GPS Kalman filter updates ina-row without any measurement rejections

(b) Continuous $64 \mathrm{~Hz}$ inertial track loop aiding reported by the in the receiver output status message

The allowable time period for GPS steering loop closure was limited to second stage ignition through third stage burnout. In addition to the pressure sensitivity of the receiver clock during climb-out there were other reasons for limiting the time window. Foremost was to avoid any attempts to force large corrections into the PBV flight path during the time period where targets were to be deployed. Additionally, since the PBV has limited divert capability, if the required correction is too large it might not be able to execute the maneuver within the available time and fuel budget. Thirdly, the PBV was required to be at a predetermined attitude with respect to the flight path of the MSX satellite during target deployments. This could potentially be at cross purposes with a divert maneuver.

The requirements for no measurement rejections and continuous inertial track loop aiding over a significant time interval were selected based on experiences accumulated from the development activities and flight test results from two previous STARS missions. A significant amount of thought and testing went into developing and proving out this strategy. If no receiver measurements are rejected during a given update cycle it means that all (up to six) sets of PR and PR-dot were within 6-sigma of their expected values. For this to repeatedly occur over a ten second time interval the filter has to be properly converged and the receiver has to be generating accurate measurement data. For the TI GPSEM to remain in aided track mode several other aspects of the system operation have to be highly accurate. In addition to properly coordinitized $64 \mathrm{~Hz}$ acceleration and navigation state data the receiver requires the external host to provide clock bias and drift estimates valid at the most recent receiver $1 \mathrm{~Hz}$ time epoch. These data are generated by combining the instantaneous raw INS outputs with GPS filter estimates and extrapolating to the required time. The accuracy level required for the aiding data is high because it is inserted directly into the receiver carrier and code track loops. If any of these data are inconsistent with the rest of the members of the set receiver will opt to ignore the data. If inconsistent data occurs repeatedly during just a few $64 \mathrm{~Hz}$ cycles the receiver will automatically take itself out of aided track mode and use its own internally generated information to attempt to maintain signal track.

If all the criteria for closing the steering loop are met the filter control logic asserts a software flag signaling the guidance algorithm to switch to the GPS-corrected navigation solution. This same flag also disables internal filter logic which forces the filter covariances to larger values when significant numbers of measurement rejections occur. Once the steering loop is closed using GPS corrections, all subsequent receiver measurements must "fit" within 6-sigma of their expected values. Measurements not meeting this constraint are rejected by the filter control logic prior to executing a measurement update cycle.

\section{Control Loop Logic Operation In-flight}

Once activated the steering control loop logic worked as designed. At the time of activation the missile was approximately at a $45 \mathrm{deg}$ pitch angle and the shroud antenna was still selected. The control loop logic began initial checking against the constraints at $T+60$ s and

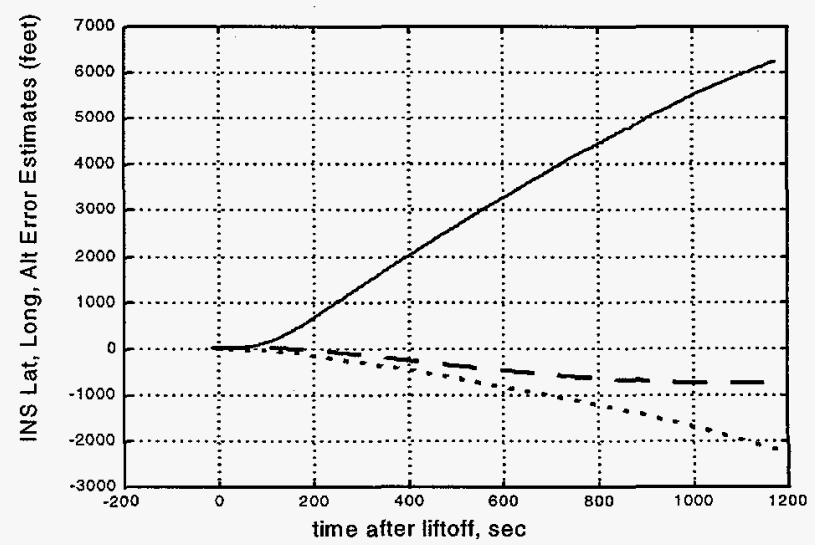

Figure 8. Plots of Error Estimates for INS latitude(solid), longitude (dashed), and altitude (dotted) from the Kalman filter 
concluded at $T+70 \mathrm{~s}$, which was the absolute earliest that it could have completed. The guidance loop was then immediately closed around the GPS-corrected navigation solution with no adverse effects. Receiver track loop aiding was continuous throughout this interval and no receiver measurement data were rejected.

\section{In-Flight GPS Filter Performance}

Figures 8 and 9 illustrate the error estimates for the three components each of INS position and velocity. Latitude and north velocity errors are noticeably larger than the longitude/east-velocity and altitude/downvelocity pairs. Pre-flight covariance analysis predicted this would occur due to gyrocompassing inaccuracy $(0.35 \mathrm{deg}$, 1-sigma). Since the trajectory was predominately to the west, the major apportionment of associated navigation error occurred in the north channel of the INS. Overall the net RSS position/velocity performance of the INS matches the pre flight covariance analysis prediction very well, although the north components are a little larger than predicted, while the east and vertical components are substantially smaller than predicted.

Figure 10 illustrates the filter estimates for the three components of INS attitude errors.

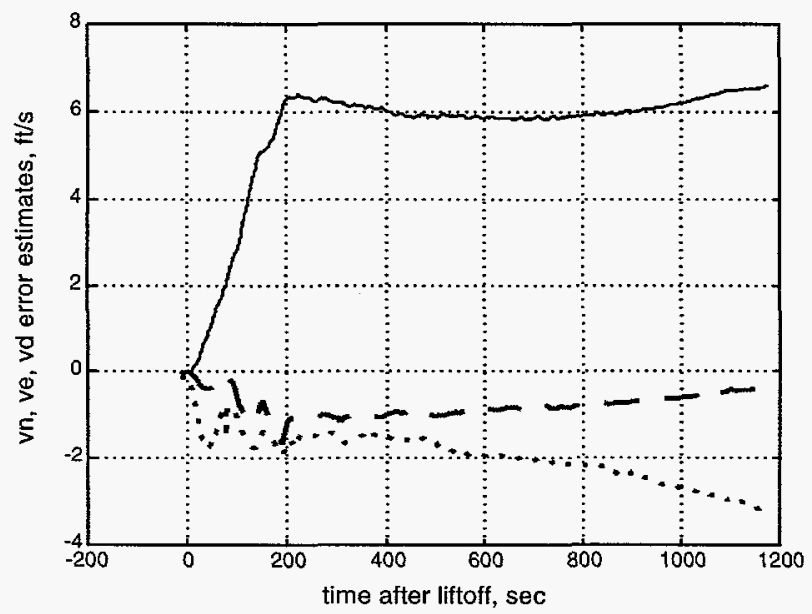

Figure 9. Plots of error estimates for INS north (solid), east (dashed), and altitude (dotted) velocity errors from the Kalman filter in $\mathrm{ft} / \mathrm{s}$

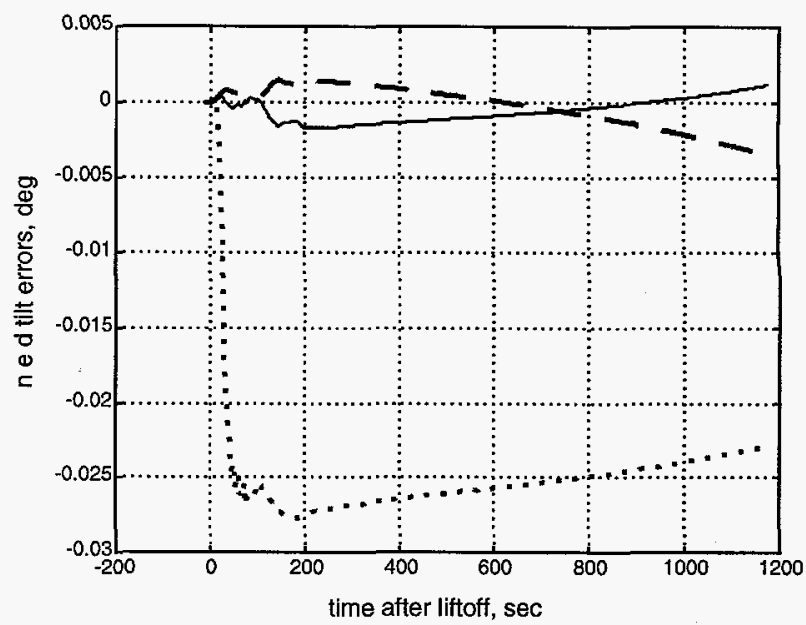

Figure 10. Plots of error estimates for INS north (solid), east (dashed), and down (dotted) attitude errors from the Kalman filter in degrees

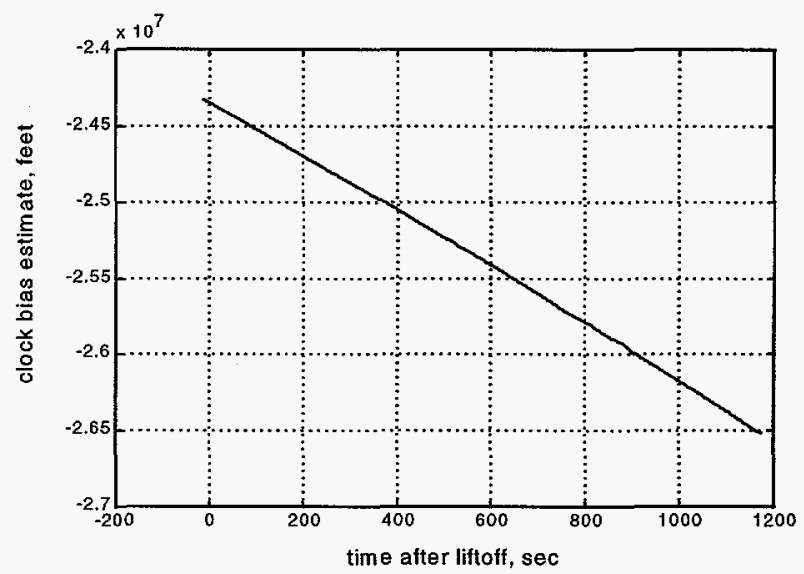

Figure 11. Plot of the receiver clock bias estimate multiplied by the speed of light, in feet

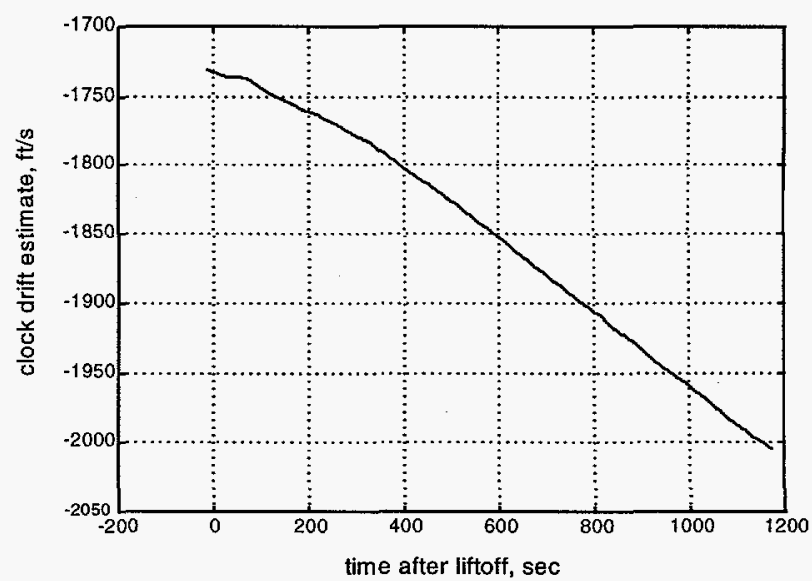

Figure 12. Plot of the receiver clock drift estimate multiplied by the speed of light, in $\mathrm{ft} / \mathrm{s}$ 
All three estimates remained very small until the missile began its dog-leg turn around the island of Niihau. Until that time no attitude states were observable. The tilt errors for the north and east axes are determined by the accuracy of the RLGA accelerometers, whereas the down axis tilt error is mainly a function of gyro accuracy and is typically expected to be an order of magnitude bigger (0.35 deg typical).

Clock bias and drift estimates are shown in Figures 11 and 12 . Both of these estimates have well behaved trends and do not exhibit any large unexpected transients as experienced on earlier flights. The magnitudes of the estimates are consistent with laboratory observations for this and other types of receivers possessing non-oven controlled oscillators. The slight flat spot in the drift rate estimate during the first $60 \mathrm{sec}$ of flight is believed to be caused by microphonic effects on the crystal oscillator caused by rapid ambient pressure changes which occur as the missile ascends through the atmosphere.

Figures 13 and 14 illustrate the filter estimates for the derivative of clock drift (clock acceleration) and g-sensitive clock drift. The clock acceleration term exhibited its largest change per unit time during ascent, then another significant change during the third stage burn and PBV deployment phase. Subsequently the clock acceleration estimate remained fairly consistent. At no time was

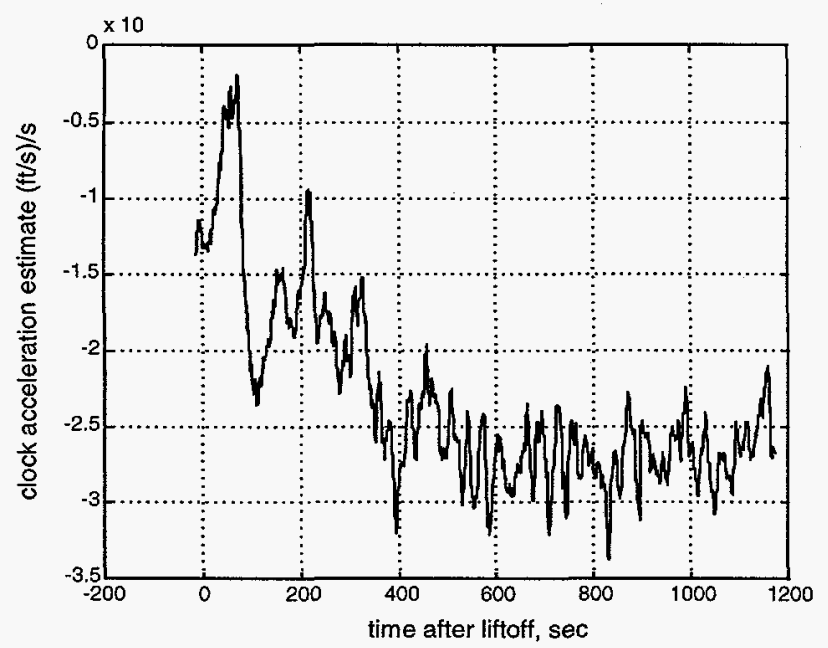

Figure 13. Filter estimate for the receiver clock acceleration (drift-dot) in (ft/s) per second

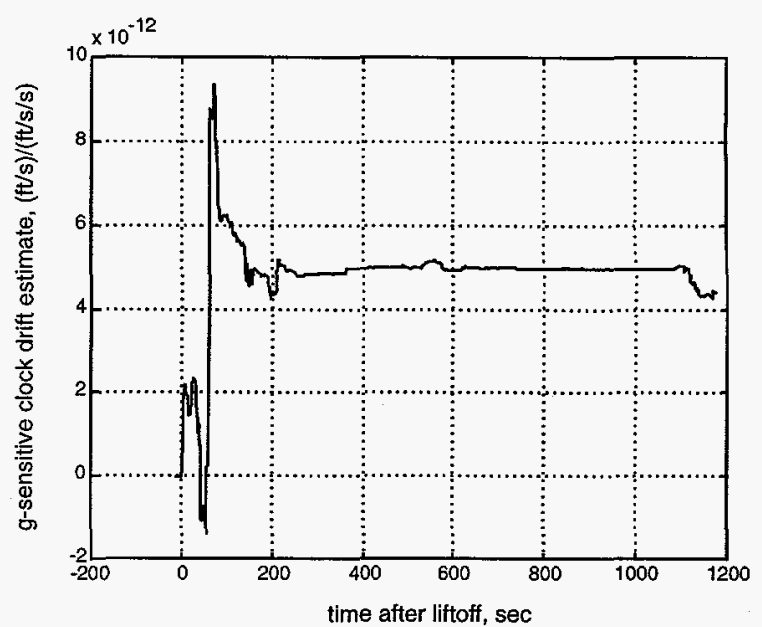

Figure 14. Filter estimate for the receiver clock gsensitive drift in (ft/s) per foot per second squared

the magnitude of the clock acceleration estimate large. This result along with other similar ones from previous flights suggests that this estimate could potentially be eliminated if computer throughput were to ever become an issue.

The g-sensitive clock drift estimate shows some activity during climb out which is likely due to clock pressure sensitivity effects. The large step change at about $60 \mathrm{~s}$ coincides with second stage ignition. By the end of the second stage motor burn this estimate acquired it's final value and did not change noticeably during the third stage burn nor during the PBV portion of the mission.

The filter rejected very few measurements during the entire period of operation. The number of measurements processed per filter cycle is illustrated in Figure 15 along with the number of SV's tracked by the receiver. The filter typically processes both PR and PR-dot measurements for a given channel, so the number of measurements processed is usually double the number of SV's tracked. The filter logic does allow for a PR measurement to be processed by itself without the companion PRdot measurement (which occurs very rarely in practice), but, not vise versa. 


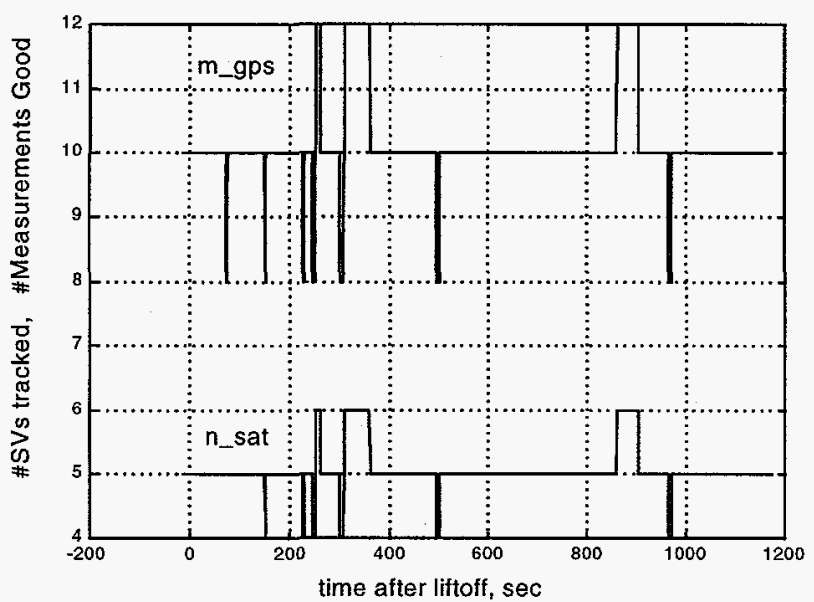

Figure 15. Comparison of the number of SV's tracked and number of measurements processed

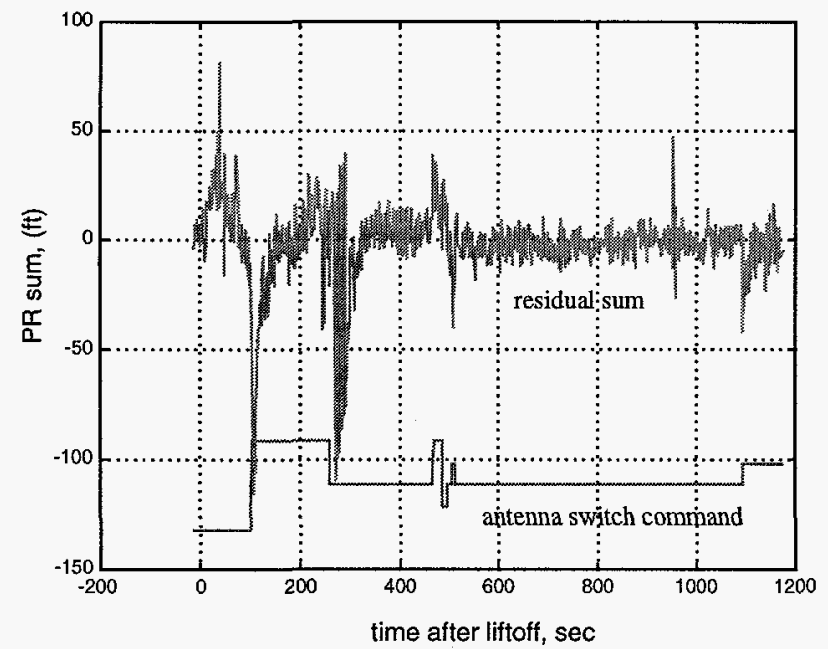

Figure 16. Sum of filter PR residuals in feet

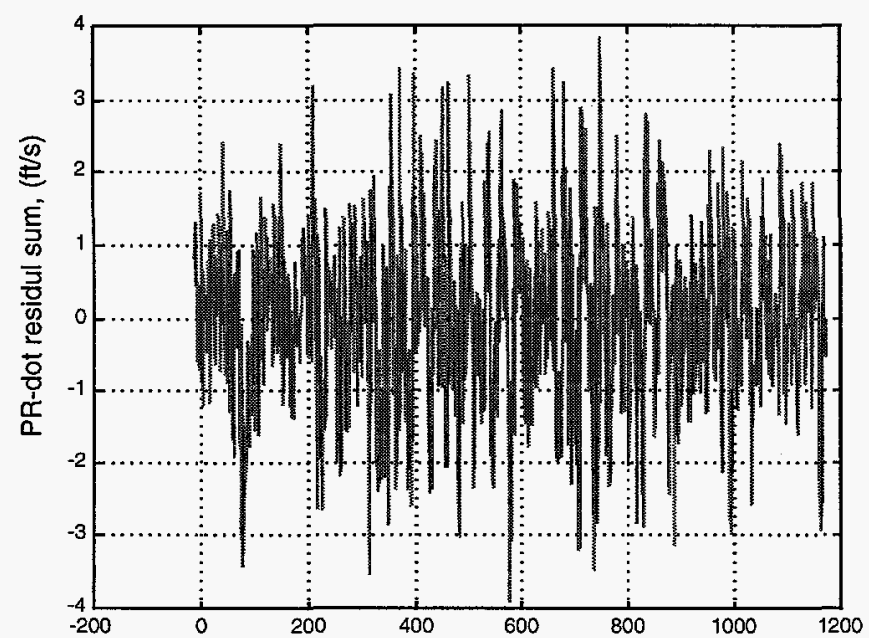

Figure 17. Sum of filter PR-dot residuals in $\mathrm{ft} / \mathrm{s}$
The sum of PR and PR-dot residuals computed by the filter are shown in Figures 16 and 17 (individual plots for each receiver channel residual are not shown to avoid classification issues). The plot of the PR residuals shows four distinct transients, each of which occurred just after switching from one patch antenna element to another. The antenna switch command has been superimposed on the PR sum plot to illustrate the timing relationship between the transients and antenna switching and is not to scale. Differences in antenna patterns and receiverto-antenna cable lengths are speculated to be the cause for the transients.

The RMS values for all PR and PR-dot filter residuals are listed by receiver channel in Table 3. The overall RMS values for PR and PR-dot residuals are $7.4 \mathrm{ft}$ and $0.27 \mathrm{ft} / \mathrm{s}$ respectively.

Table 3. PR and PR-dot Residual Statistics for each GPSEM Channel

\begin{tabular}{|c|c|c|c|c|c|}
\hline $\begin{array}{c}\text { Psuedo } \\
\text { Rang }\end{array}$ & $\begin{array}{c}\text { MEAN } \\
(\mathrm{ft})\end{array}$ & $\begin{array}{c}\text { RMS } \\
(\mathrm{ft})\end{array}$ & $\begin{array}{c}\text { Range } \\
\text { Rate }\end{array}$ & $\begin{array}{c}\text { MEAN } \\
(\mathrm{ft} / \mathrm{s})\end{array}$ & $\begin{array}{c}\text { RMS } \\
(\mathrm{ft} / \mathrm{s})\end{array}$ \\
\hline PR1 & -0.1 & 8.9 & PRR1 & -0.27 & 0.27 \\
\hline PR2 & +1.7 & 4.4 & PRR2 & -0.01 & 0.22 \\
\hline PR3 & -0.3 & 4.7 & PRR3 & +0.02 & 0.26 \\
\hline PR4 & -1.2 & 6.6 & PRR4 & +0.01 & 0.27 \\
\hline PR5 & -2.8 & 7.4 & PRR5 & -0.01 & 0.27 \\
\hline PR6 & -3.1 & 12.2 & PRR6 & -0.00 & 0.28 \\
\hline
\end{tabular}

Ground Tracking Radar Comparisons

The three components of position error from the GPS Kalman filter are compared against like measurements derived from PMRF ground based tracking radar in Figures 18,19, and 20. These plots are included for the specific purpose of impressing the stalwarts in the audience who cling to the idea that tracking radar is always better than GPS.

The radar data plotted represents the difference between the post-processed radar position solution in the geographic frame and the three components of position outputs from the inertial navigation system. Prior to liftoff the tracking radar had significant errors for all three components as compared to GPS. Early in the flight the radar data agreed well with the GPS solution, although there are notable large transients in the radar data at 


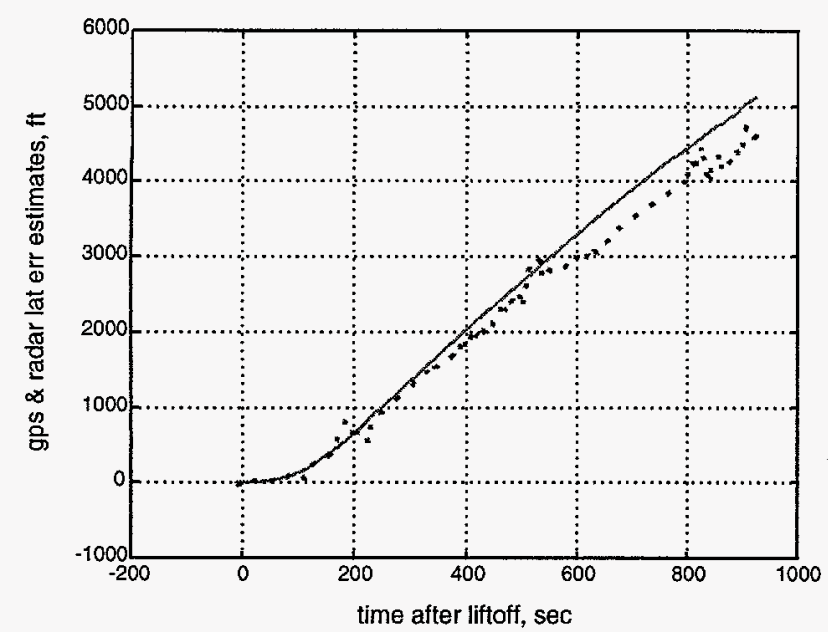

Figure 18. Comparison of latitude errors from GPS (solid) and ground radar (dotted) in feet

200 sec which is where the PBV deployed and executed a large attitude maneuver. As the mission proceeded the radar and GPS answers gradually diverged which reflects the fact that the radar solution becomes less accurate as the measurement distance increases. Overall the GPS data was much smoother and consistent than the radar. GPS data was readily available in real time and was trivial to reduce and analyze after the flight test compared to the effort required to reduce the radar data. Based on the PR residual data previously presented, the GPS position solution is clearly more accurate than the position solution obtained from the radar.

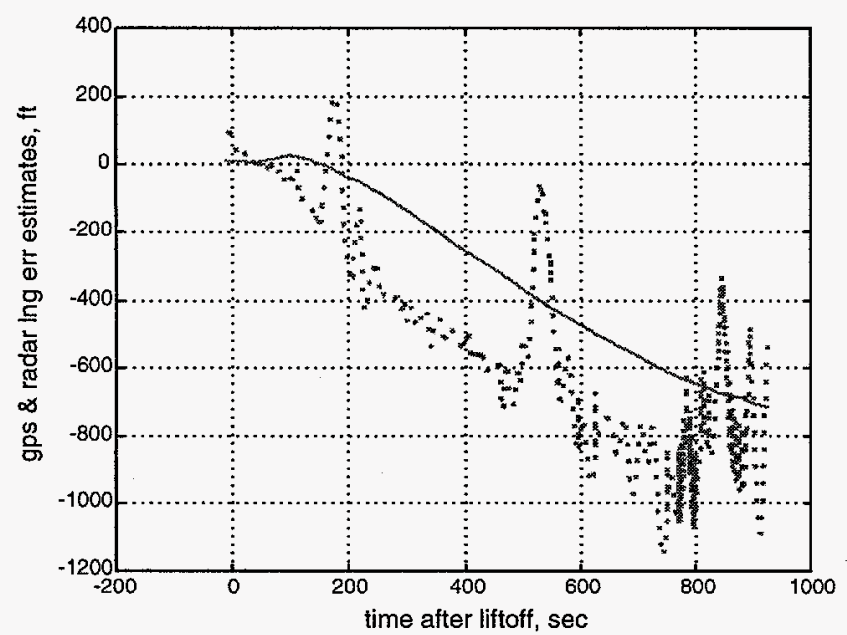

Figure 19. Comparison of longitude errors from GPS (solid) and ground radar (dotted) in feet

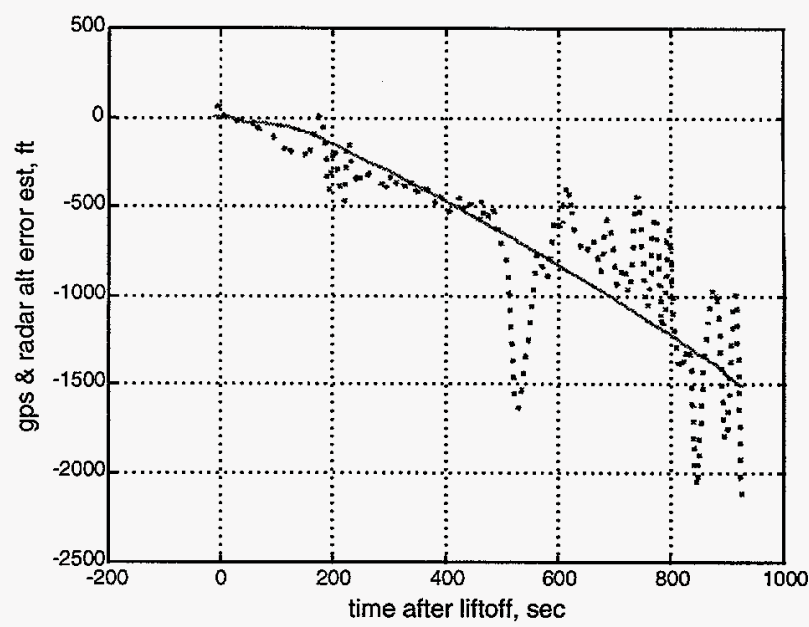

Figure 20. Comparison of altitude errors from GPS (solid) and ground radar (dotted) in feet

\section{Conclusions}

Overall the GPS system performance achieved on this mission was the best ever experienced by a STARS missile. The tracking performance of the receiver was notable both in quantity (number of SV's tracked) and quality (overall PR and PR-dot residual values). The general overall performance of GPS subsystem was better due to: (1) the sealed enclosure used to mitigate the clock pressure sensitivity effects; (2) improvements made to the antenna system; and (3) past experience that was incorporated back into the GPS control software design.

The software installed for this mission dedicated to closing the steering loop around the GPS-aided navigation solution worked as intended. Once the steering loop was closed the guidance system maintained the missile trajectory very close to preflight nominal.

The availability of the GPS-aided navigation solution proved useful for both guiding the missile and also for generating the pointing angles for a vast array of downrange ground and airborne sensors. Aided with GPS, the STARS PBV delivered the MDT-II targets at the designated positions and times and the MSX satellite sensors were then able to acquire and view them as designed. The end result is that a wealth of satellite and downrange sensor data were obtained. 


\section{Acknowledgments}

Sandia is indebted to the STARS Product office for providing the opportunity and financial support for the STARS GPS initiative. The author wishes to personally recognize and thank Dr. Alfred C. Watts, Sandia National Laboratories, for the development and implementation of the GPS STARS Kalman filter software. Thanks also to the many individuals in SNL departments 2334, 2425, and 2526 who's collective efforts have made the STARS program in general, and the navigation, guidance, and control subsystems in particular, resounding successes.

\section{References}

[1] A. Watts, R. Eno, H. Wente, and C. Curtis, "Strategic Target System (STARS) Launch Vehicle", AIAA Missisle Sciences Conference, Monterey, CA, Nov 1988.

[2] A. Watts, "Pulse Mode Control of the ODES Post Boost Vehicle", AIAA /BMDO Technology Readiness Conference, San Diego, CA, Aug 1997.

[3] B. Sims and J. White, "The STARS Launch of the MSX Dedicated Target, MDT-II", AIAA/BMDO Technology Readiness Conference, San Diego, CA, Aug 1997.

[4] C. Borgman and L. Dalton, "An Embedded Multiprocessor Computer for Proof-ofPrinciple Testing of Exploratory Systems Concepts", AIAA Computers in Aerospace VI Conference, Wakefield, MA, Oct 1987.

[5] M. Weber and M. Robinson, "Development of the STARS Composite Payload Fairing", $42^{\text {nd }}$ International SAMPE Symposium, Anaheim, CA, May 1997.

[6] J. White, "Guidance and Targeting for the Strategic Target System", AIAA Guidance, Navigation, and Control Conference, DISCLAIMER Portland, OR, Aug 1990.

This report was prepared as an account of work sponsored by an agency of the United States Government. Neither the United States Gnvernment nor any agency thereof, nor any of their employees, makes any warranty, express or implied, or assumes any legal liability or responsibility for the accuracy, completeness, or usefulness of any information, apparatus, product, or process disclosed, or represents that its use would not infringe privately owned rights. Reference herein to any specific commercial product, process, or service by trade name, trademark, manufacturer, or otherwise does not necessarily constitute or imply its endorsement, recommendation, or favoring by the United States Government or any agency thereof. The views and opinions of authors expressed herein do not necessarily state or reflect those of the United States Government or any agency thereof. 


\section{DISCLAIMIER}

Portions of this document may be illegible in electronic image products. Images are produced from the best available original doccoment. 